\title{
UNIMODALITY OF CERTAIN PARAMETRIC INTEGRALS
}

\section{IOSIF PINELIS}

Abstract. The unimodality of the functions represented as certain parametric integrals is considered. A crucial part of the proof is based on Kemperman's necessary and sufficient condition for the unimodality of all mixtures of a given family of functions.

Mathematics subject classification (2010): Primary 26A48, 26D15; Secondary 26A51, 47G10.

Keywords and phrases: Unimodality, mixtures, parametric integrals, convex functions, concave functions, monotonicity.

\section{REFERENCES}

[1] Unimodality of a certain parametric integral, 2015, MathOverflow, http://mathoverflow.net/ questions/209072/unimodality-of-a-certain-parametric-integral/209481\#209481.

[2] S. Dharmadhikari And K. Joag-Dev Unimodality, convexity, and applications, Probability and Mathematical Statistics, Academic Press Inc., Boston, MA, 1988.

[3] J. H. B. Kemperman, Mixtures with a limited number of modal intervals, Ann. Statist., 19 (4): 2120-2144, 1991. 